\section{Deutsche Akkreditierungsstelle GmbH}

\section{U. Zimmermann}

Gesundheit/Forensik, Deutsche Akkreditierungsstelle GmbH (DAkkS), Frankfurt, Deutschland

\section{Synonym(e) DAkkS}

\section{Englischer Begriff German Accreditation Body}

Beschreibung Die Deutsche Akkreditierungsstelle GmbH (DAkkS) ist die nationale Akkreditierungsstelle der Bundesrepublik Deutschland. Sie entstand auf Grundlage der Verordnung (EG) Nr. 765/2008 und des Akkreditierungsstellengesetzes (AkkStelleG) und nahm am 1. Januar 2010 ihre Arbeit auf.

Um ihre hoheitlichen Akkreditierungsaufgaben ausfüllen zu können, wurde die DAkkS vom Bund beliehen. Als beliehene Stelle untersteht die DAkkS der Aufsicht des Bundes und ist nicht gewinnorientiert ausgerichtet. Gesellschafter der GmbH sind zu gleichen Teilen die Bundesrepublik Deutsch- land, die Bundesländer und die durch den Bundesverband der Deutschen Industrie e. V. (BDI) vertretene Wirtschaft.

Als unabhängige Stelle begutachtet, bestätigt und überwacht die DAkkS in allen Bereichen der Wirtschaft die Fachkompetenz insbesondere von Laboratorien, Zertifizierungsund Inspektionsstellen. Zu diesen Laboratorien gehören auch medizinische Laboratorien, die auf Grundlage der Norm DIN EN ISO 15189 akkreditiert werden. Im Bereich der Akkreditierung von medizinischen Laboratorien ist die DAkkS eine der führenden Akkreditierungsstellen in Europa.

Sie ist Mitglied der internationalen Akkreditierungsorganisationen European co-operation for Accreditation (EA), International Accreditation Forum (IAF) und International Laboratory Accreditation Cooperation (ILAC) und Unterzeichnerin der multilateralen Abkommen dieser Organisationen. Dadurch werden Akkreditierungen der DAkkS weltweit anerkannt.

\section{Literatur}

www.dakks.de. Zugegriffen am 23.05.2018 